\title{
Double-Layer Grid Filter for Control of Wireless Signal Propagation within Buildings
}

\author{
P. Callaghan, R. Huelin, Md Hasanuzzaman Sagor \\ University of Greenwich \\ Faculty of Engineering and Science \\ Chatham Maritime, Kent ME4 4TB, UK
}

\begin{abstract}
A simple Double-Layer Grid plane wave filter structure is proposed that can provide multiple transmission bands for cellular phone frequencies but with a reflection band for WLAN signals. The approach offers ease of construction making it applicable to building applications. A parametric study using simulation supported by simple experimental data investigates the proposed, novel design.
\end{abstract}

Keywords-Antennas, Frequency Selective Surfaces, Spatial Filtering

\section{INTRODUCTION}

The increase of wireless systems over the last couple of decades has resulted in overcrowding in the electromagnetic spectrum. System designers have developed novel modulation techniques and multi-antenna solutions, such as MIMO, to increase data capacity over a given wireless channel. Perhaps the greatest challenge is presented by the modern office building, wherein there is a high density of users. Propagation through the structure of the building structure itself has to be mitigated and some interesting solutions are presented in [1] particularly with regard to steel reinforced materials. However, a major challenge in the design of the building is to permit 'macro-cell' communications (i.e. mobile phone) to propagate through the whole structure, but to limit propagation of internal wireless LAN to a specific area, such as a single office, allowing re-use of the WLAN band in adjacent rooms [2]. To this end much research has been carried out into the application of Frequency Selective Surface (FSS) technology to the design of the modern building - the aim being to restrict wireless propagation to within a 'room', by making the walls act as a plane wave filter [3].

Research into FSS design for buildings [3] has focused principally on realizing novel element structures to meet the wide frequency bandwidths involved - control is needed from $400 \mathrm{MHz}$ (for TETRA mobile radio) up to $2.4 \mathrm{GHz}$ (WLAN frequencies), demanding a 6:1 frequency response. The upper 'useful' frequency of an FSS is usually limited by the appearance of 'trapped waves' [4] as the periodicity approaches a wavelength. Most researchers have limited themselves to investigating novel single layer FSS structures [5]. Some work has been undertaken into double-layer structures - although to improve the band shape provided by a single-layer resonance. In [3] two cascaded FSS were demonstrated to widen the bandwidth of the resonance whilst some workers have used two different patterns in close proximity to generate FSS with a wide band spacing [6]. Fabrication has also been a significant challenge and some recent work has proposed solutions for integrating FSS onto glass [7] (for windows and partitions) and even blinds [8]. The double-layer design on glass in [7] is of interest as it offers a double-resonance response, similar to the work in this paper, but using a different approach. Naturally propagation within a building is also dependent on scattering and an insight into this has been given in [9] which investigates a wall made using 'Smart bricks' employing FSS.

Fabrication of FSS is a significant challenge, and many of the structures proposed would be difficult to build on a large scale - certainly to cover walls, floors and ceilings. Rather this paper re-visits the problem allowing transmission of waves through steel reinforced material in buildings and look to see if can take advantage of the steel reinforcement, rather than consider it a drawback, as in [2]. It is well known that cascading 2 or more gratings can generate transmission bands [10]. We review this approach and investigate two embedded metallic meshes, as illustrated in Fig. 1, and tune their plane wave transmission line effects to create passbands. Note one or more of these grids could be a metallic reinforcing mesh. For this work we took a design goal of passing cellular radio signals at either $\sim 900 \mathrm{MHz}$ or $\sim 1800 \mathrm{MHz}$ but with an option to reflect WLAN $(2.4 \mathrm{GHz})$ signals.

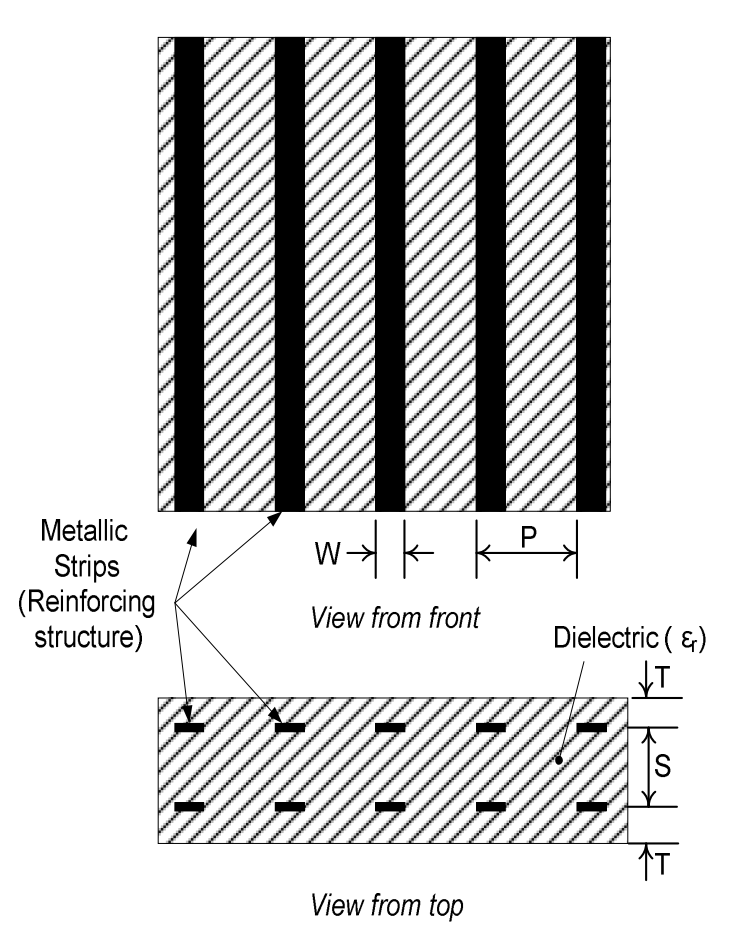

Fig. 1: Outline of proposed Double-Layer Grid Filter 


\section{DOUBLE LAYER GRID FILTER DESIGN}

The Double-Layer Grid Filter structure is presented in Fig.1. It consists of 2 strip gratings, or grids, separated in space by a dielectric former. It is well known that a strip grating can be modelled as an inductance in an equivalent circuit representation of a plane wave structure [11]. Here the electric field of the incident wave must be parallel to the wires; hence they reflect the wave, with an 'inductive' effect. At low frequencies, defined by the spacing between the wires being much smaller than the wavelength, the grating starts to act like a reflector and no signal passes through - hence providing 'screening' at lower frequencies blocking wireless signals, as discussed in [2].

Note in this paper strip gratings are considered, but the results would apply equally well if a mesh is used - the grating here are linearly polarized, whilst a mesh would be used to provide a dual-polarized FSS. Similarly we assume infinitely thin, flat conductors - but finite thickness, or even round wires will give similar results.

Using the transmission line analogy, as illustrated in Fig.2, it is easily understood that a second grating placed approximately one quarter of a wavelength away causes a resonance between the two layers. The inductance of the second grating is transformed to be capacitive at the plane of the first grating, creating a parallel resonant circuit having a transmission band. The frequency of the resonance is determined by the inductance of each grid and the spacing between them, so a transmission line circuit simulator was used initially to calculate suitable values. It was also expected that a second passband would be formed, likely at 3 times the frequency of the first resonance (i.e. when the spacing was three-quarters of a wavelength). However, the transmission line simulator calculated that the frequency of the second resonance could be much lower, nearer only twice that of the first resonance. For example, the frequency response of a circuit with two $2 \mathrm{nH}$ inductors separated by a transmission line of impedance $50 \mathrm{Ohm}$, and length $130 \mathrm{~mm}$ is shown in Fig. 3. This provides transmission bands around $1 \mathrm{GHz}$ and at $2 \mathrm{GHz}$, to suit the cellular mobile phone bands. This structure is equivalent to placing two grids $130 \mathrm{~mm}$ apart in free space. Also plotted in Fig. 3 is the transmission response with a single inductor of value $1 \mathrm{nH}$ (i.e. the $2 \times 2 \mathrm{nH}$ in parallel) illustrating a transmission loss of around $14 \mathrm{~dB}$ at $1 \mathrm{GHz}$, and much higher at lower frequencies. Clearly using two grids rather than one can resolve 'screening' problems, for specific bands.

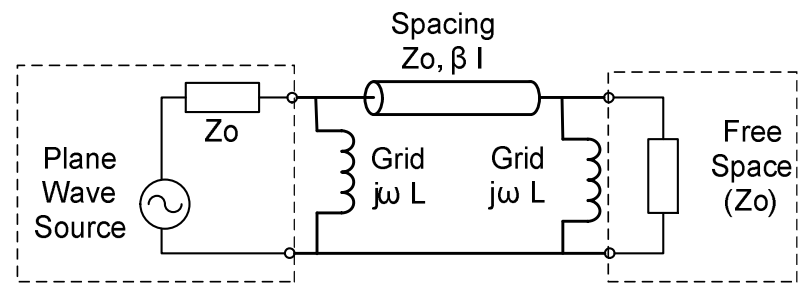

Fig. 2: Equivalent circuit representation of Double-Layer Grid Filter

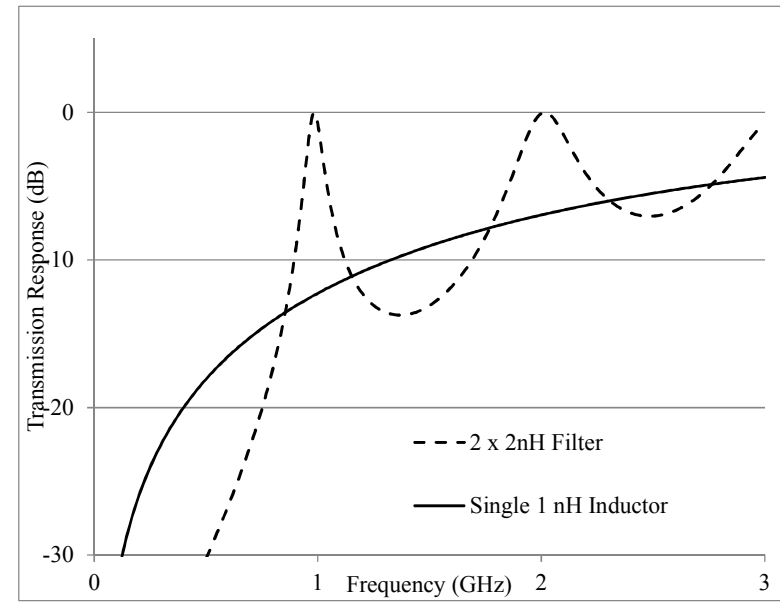

Fig. 3: Frequency Response of Transmission Line Filter Circuit

Whilst a 'passband' has been achieved at $1 \mathrm{GHz}$ in Fig.3 the bandwidth is quite narrow. Currently the UHF mobile phone bands are being extended significantly (i.e. for LTE) such that this tuned passband may have limited applicability. However, the band width can be altered by adjusting the parameters of the transmission line circuit. To investigate this 4 structures were tuned to $\sim 900 \mathrm{MHz}$ using different values of inductance and transmission line length, as give in Table 1. The frequency responses of these four different designs are compared in Fig 4.

Table 1: Details of Transmission Line Filter in Fig. 4

\begin{tabular}{|c|c|c|}
\hline $\begin{array}{c}\text { Inductor Value } \\
(\mathbf{n H})\end{array}$ & $\begin{array}{c}\text { Transmission Line } \\
\text { Length }(\mathbf{m m})\end{array}$ & $\begin{array}{c}\text { Bandwidth at } \\
\mathbf{8 0 0} \mathbf{~ M H z}\end{array}$ \\
\hline 1 & 175 & $1 \%$ \\
\hline 2 & 160 & $5 \%$ \\
\hline 5 & 130 & $30 \%$ \\
\hline 10 & 110 & $60 \%$ \\
\hline
\end{tabular}

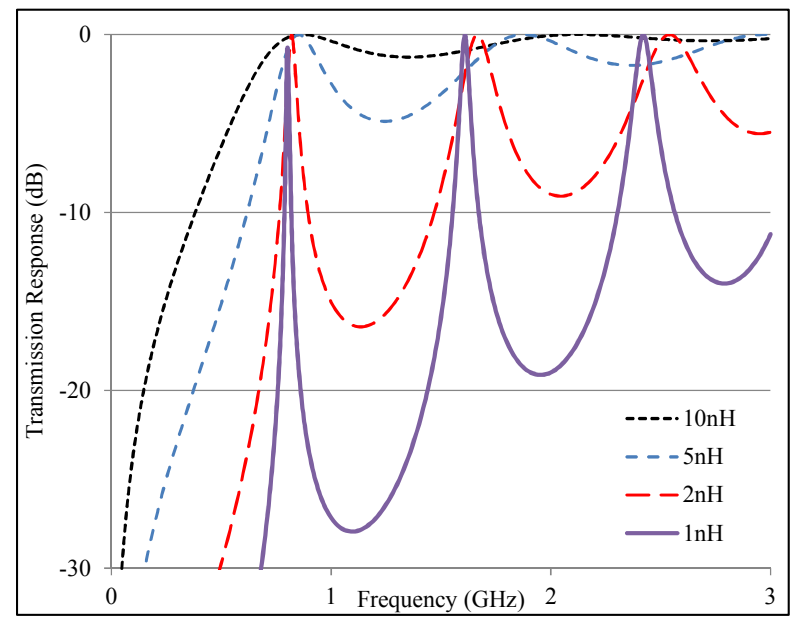

Fig 4: Transmission Line Filters using different Inductances

It is seen that by increasing the inductance the bandwidth increases. Indeed for $10 \mathrm{nH}$ the bandwidth is so wide that the bands begin to merge, and form a low pass structure. Rather 
than a single metallic reinforcement causing 'screening' and loss of signal, a double layer structure could provide multiple resonances allowing good transmission of mobile signals.

\section{Plane Wave Structure}

The responses shown in Figs. 3 and 4 assume that each grid behaves like an inductor - but there are differences. At lower frequencies the variation of the grid reactance using closed form expressions [12] has been shown to be similar to an inductance provided that the ratio of $\mathrm{W}$ to $\mathrm{P}$ as defined in Fig. 1 is much less than 1 . The reactance is also very dependent upon incidence angle - this is explored further in section V. However, as the wavelength becomes smaller than the periodicity of the structure the equivalent circuit analogy breaks down. Above this point diffraction effects occur (generation of additional waves). Hence in designing a plane wave filter using two grids the periodicity of the grids ( $P$ in Fig. 1) is a critical design parameter.

An additional design goal was to achieve a reflection band at $2.4 \mathrm{GHz}$, typically needed to create isolated 'WLAN zones'. In Fig. 4 stopbands can be provided using small values of 'inductance' - but at the loss of the desired wider passbands. However it is well known that when the wavelength becomes equal to the periodicity a surface wave can be excited that results in a deep, narrow reflection band [4]. Here we propose to make the periodicity a wavelength at $2.4 \mathrm{GHz}$ (i.e. $\lambda=125 \mathrm{~mm}$ ) to deliberately generate a surface wave resonance to provide the desired reflection band. A grid structure having $\mathrm{W}=20 \mathrm{~mm}$ and $\mathrm{P}=125 \mathrm{~mm}$ was calculated to give $\sim 5 \mathrm{nH}$ inductance (using [12]) and then the double layer structure, with spacing of $130 \mathrm{~mm}$ was simulated using CST Microwave Studio. CST MWS has a Floquet mode model for infinite periodic array - this includes diffraction waves so can model a grating accurately. The simulations from CST for normal incidence are compared to the equivalent circuit model response in Fig. 5.

As can be seen from Fig. 5 there is reasonable agreement between the equivalent circuit model and a practical plane wave structure. - both giving the two passbands around 900 and $1800 \mathrm{MHz}$. However, there is a significant difference with the appearance of a surface wave null at higher frequencies. This is higher than expected, at $2.7 \mathrm{GHz}$, but clearly the structure offers a very desirable transmission / reflection characteristic.

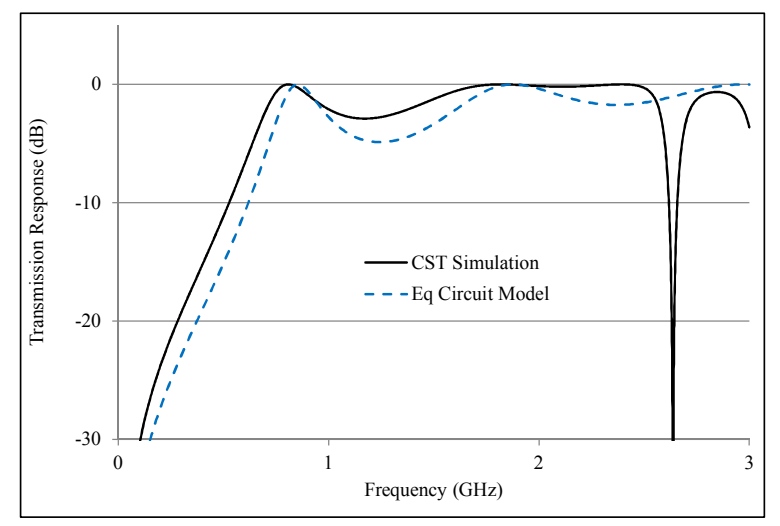

Fig 5: Comparison of Lumped Element Line Fiter to Double Layer Grid Filter

\section{EFFECT OF DIELECTRIC}

The response in Fig. 5 is for 'free-standing' gratings. In practice the gratings need to be supported by a dielectric material. CST was used again to investigate the response, at normal incidence, for a double grating structure immersed in various dielectrics. To assess the practical application of this work the permittivities to be used need to be representative of typical building materials. For this, measured values were taken from [13] where sample building materials have been characterized. For comparison the dimensions of the structure in different dielectrics were scaled to give passbands around 900 and $1800 \mathrm{MHz}$ whilst also producing a surface wave reflection band at $2.4 \mathrm{GHz}$. (Note there is a small layer of dielectric covering the gratings, such that the arrays are essentially in an infinite medium, then the dimensions simply scale by $1 / \sqrt{ } \varepsilon_{r}$, see [4]). The values of permittivity used along with the dimensions for each substrate are included in Table 2. The simulated transmission characteristics are compared in Fig. 6.

We note from Fig. 6 that embedding the gratings in a dielectric does produce a narrow passband, particularly at the lower frequency. The higher passband $(\sim 1800 \mathrm{MHz})$ exhibits additional resonances for the higher permittivity materials. However, the overall transmission loss is generally below 5 $\mathrm{dB}$ for both passbands. The surface wave null has been moved to $2.4 \mathrm{GHz}$ and has a similar shape regardless of permittivity. The transmission loss is greater than $20 \mathrm{~dB}$ but only over a very narrow bandwidth $(<10 \mathrm{MHz}-$ Wifi typically channels are $22 \mathrm{MHz}$ wide from 2.41 to $2.49 \mathrm{GHz}$ ).

\section{EFFECT OF INCIDENCE ANGLE}

The response so far has considered only normal incidence but in practice the waves may enter a room over a range of angles of incidence and so transmission responses were simulated firstly for an air-spaced structure at various TE angles of incidence. These are presented in Fig. 7. As might be expected the response changes markedly with incidence angle. The primary effect of the lower passband is seen to increase in frequency and the transmission response deteriorates considerably for incidence angles above $40^{\circ}$, which is not particularly high.

Table 2: Details of different dielectric used in study

\begin{tabular}{|l|c|c|c|c|c|r|}
\hline \multicolumn{1}{|c|}{ Material } & $\boldsymbol{\varepsilon}_{\mathbf{r}}$ & $\tan \boldsymbol{\delta}$ & $\mathbf{P}$ & $\mathbf{W}$ & \multicolumn{1}{c|}{$\mathbf{S}$} & \multicolumn{1}{c|}{$\mathbf{T}$} \\
\hline Air & 1.0 & 0.0 & 139 & 13.4 & 130 & 20 \\
\hline Dry Wall & 2.1 & - & 96.3 & 9.3 & 89.9 & 13.8 \\
\hline Plywood & 2.4 & 0.03 & 90.6 & 8.7 & 84.6 & 13.0 \\
\hline Hollow Concrete & 3.5 & 0.30 & 74.9 & 7.2 & 69.9 & 10.8 \\
\hline Brick & 5.1 & 0.04 & 61.7 & 5.9 & 57.6 & 8.9 \\
\hline Solid Concrete & 7.7 & 0.19 & 50.1 & 4.8 & 46.8 & 7.2 \\
\hline
\end{tabular}




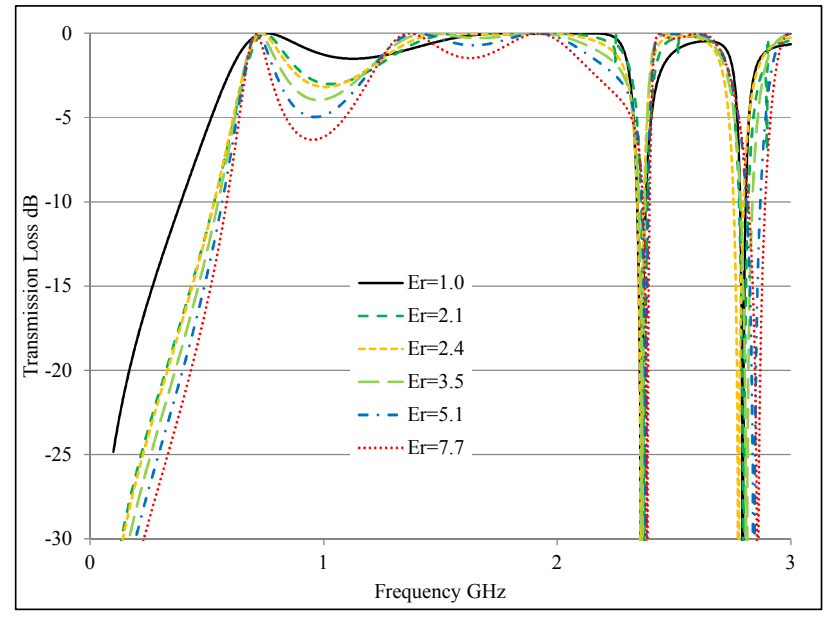

Fig. 6: Effect of Dielectric on Grid Filter Response (Normal Incidence)

Importantly the $2.4 \mathrm{GHz}$ surface wave resonance exhibits significant changes. Firstly the frequency of the null reduces with incidence angle (as might be expected from [11]). There also appears to be multiple resonances forming above the first surface wave null. Ultimately the surface wave null has a detrimental affect overall. The simple air-spaced structure therefore has limited application.

It is well known that a dielectric can reduce the effects of incidence angle of an FSS structure [4] and the intention for this structure is that it should be embedded in a wall structure - indeed may even constitute 'reinforced concrete'. Further simulations were made for the structure in a dielectric of permittivity 7.7, using the dimensions from Table 2. The transmission response is plotted in Fig. 8.

It can be seen from Fig. 8 that the effect of incidence angle is reduced, hence curves are plotted to higher incidence angles $\left(60^{\circ}\right)$. The lower passband is now seen to be stable with change of incidence angle. However, the bandwidth does reduce considerably with up to $10 \mathrm{~dB}$ transmission loss being introduced around 1.2 GHz. Similar effects are seen at the higher transmission bands. The shift in the surface wave

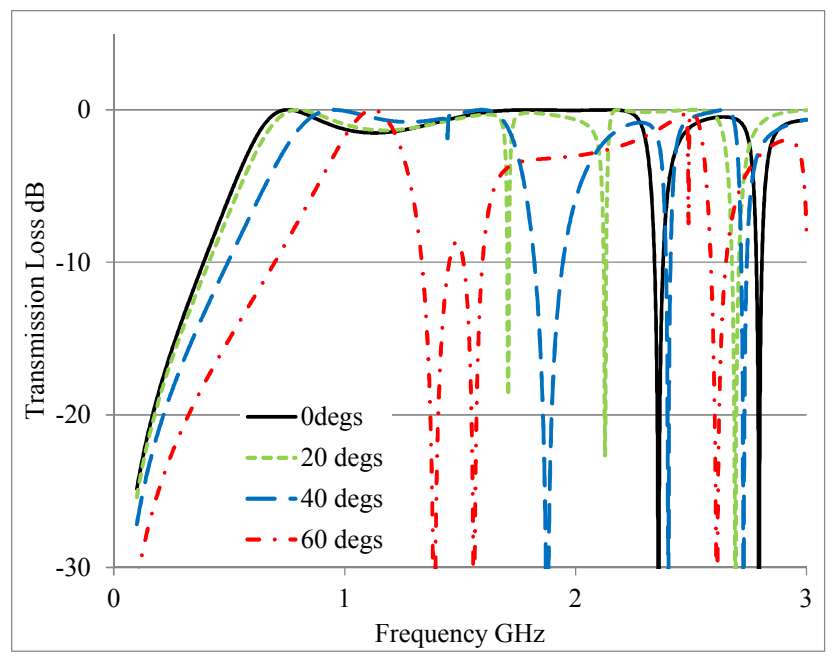

Fig. 7: Transmission Response at different incidence angles for air-spaced double grid filter

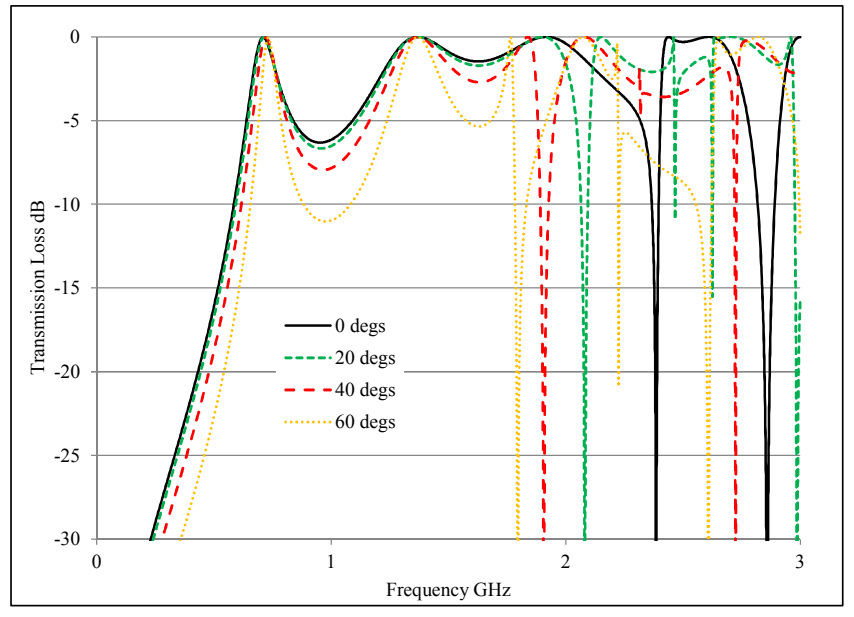

Fig. 8: Transmission Response for different incidence angles for Double Layer Grid Filter embedded in a 'Solid Concrete' $\left(\varepsilon_{\mathrm{r}}=7.7\right)$

frequency of the surface wave resonance changed by $35 \%$ but only $24 \%$ for the embedded structure. Unfortunately this is still significant with nulls appearing below $2 \mathrm{GHz}$.

\section{EXPERIMENTAL DOUBLE LAYER GRID FILTER}

To evaluate the proposed structure a prototype was constructed using the initial air-spaced dimensions of $\mathrm{W}=20 \mathrm{~mm}, \mathrm{P}=125 \mathrm{~mm}$ and $\mathrm{S}=130 \mathrm{~mm}$. The grids were constructed by fixing copper tape onto $5 \mathrm{~mm}$ thickness Perspex panel of overall size $1 \mathrm{~m} \times 1 \mathrm{~m}$. The two panels were held apart using plastic screws, and mounted on a wooden post for measurement. The experimental hardware is shown in Fig. 9.

The panel was held in the far field of a wideband horn, such that the incident radiation was essentially a plane wave. However we were restricted to using smaller broadband antennas close to the sample - essentially probing the 'near field' and as the sample was of finite size this caused some problems. The measured 'plane wave' transmission response for $10^{\circ}$ incidence is presented in Fig. 10 (dashed line) for comparison to the expected response simulated using CST assuming infinite arrays.

The measured response clearly differs from the ideal, simulated response in Fig. 10 particularly at low frequencies. However much of this can be attributed to the finite size of the prototype. For instance, at $600 \mathrm{MHz}$ the strips are a halfwavelength long, behave like dipoles, and there is evidence of a null. Conversely at $900 \mathrm{MHz}$ the strips are three quarters of a wavelength and can have an 'anti-resonance' - here seen to focus the waves and create 'gain'. As the frequency increases they start to approximate the 'inductance' and there is similarity to the simulated infinite array. At higher frequencies a reflection null is measured around $2.1 \mathrm{GHz}$ - evidence of a surface wave effect. The width of this reflection band appears larger than predicted by the computer model; however it is less well defined having a depth of only $20 \mathrm{~dB}$. 


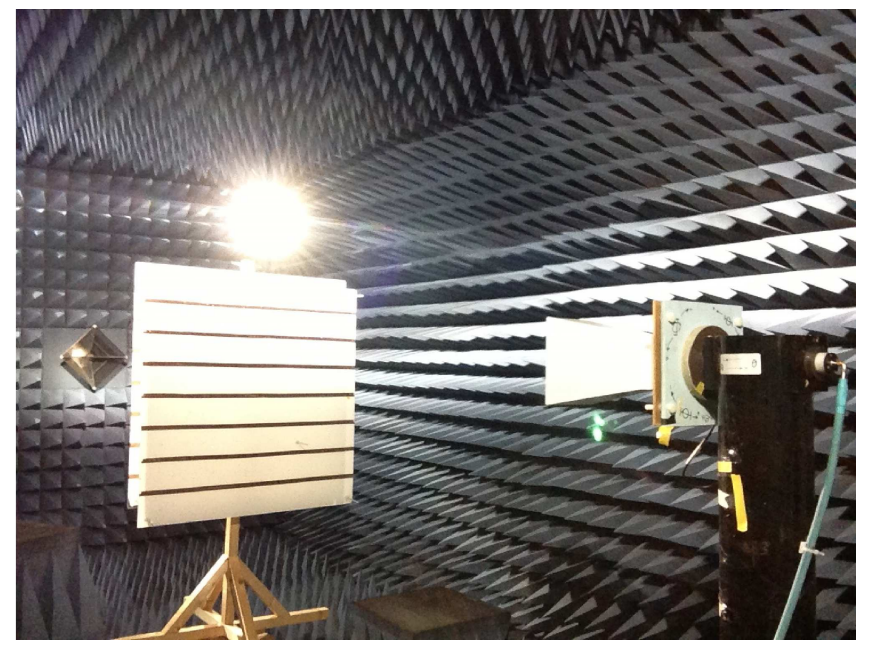

Fig. 9: Photo of Experimental Double-Layer Grid Filter under Test

\section{CONCLUSION}

It has been shown that introducing a second wire mesh into a wire loaded material can provide transmission bands, offering a solution to the problem of obstruction of wireless signals by reinforced building materials. Here 2 free standing gratings were shown to provide transmission bands at 900 $\mathrm{MHz}$ and $1800 \mathrm{MHz}$. Embedding these gratings in a dielectric gave two benefits. Firstly transmission properties of the dielectric itself can add additional transmission bands, offering transmission to most mobile bands. Secondly the presence of a dielectric reduced the effect of incidence angle. The structure here was kept symmetrical so further band shaping may be possible using asymmetry. In particular this work hasn't considered provision of a lower band at $400 \mathrm{MHz}$ for emergency services, but the multi-resonances achieved with embedded dual-layer grids indicated this could be provided for,

Surface wave nulls are expected with periodic structures but in this work the use of this phenomenon was considered to provide reflection bands to contain WiFi /WLAN signals at $2.45 \mathrm{GHz}$. This was demonstrated in principle, but in practice the surface wave null has too narrow a bandwidth and also was found to be too sensitive to angle of incidence, even in the presence of a dielectric.

The challenge of controlling propagation in buildings fundamentally rests in presenting a solution that can be realized. Steel reinforced concrete is a common building material and taking this dual-grating approach may lead to a practical solution.

\section{REFERENCES}

[1] A. Newblold: 'Designing buildings for the wireless-age', IEE Communications Engineer, Vol. 2, Iss. 3, pp 18-21

[2] M Philippakis, C Martel, D Kemp: 'Application of FSS structures to selectively control the propagation of signals into and out of buildings Annex 4: In-building propagation enhancement' Ofcom ref. AY4464A,
2004 [Online] Available: http://stakeholders.ofcom.org.uk/binaries/research/spectrum-research/in_building.pdf

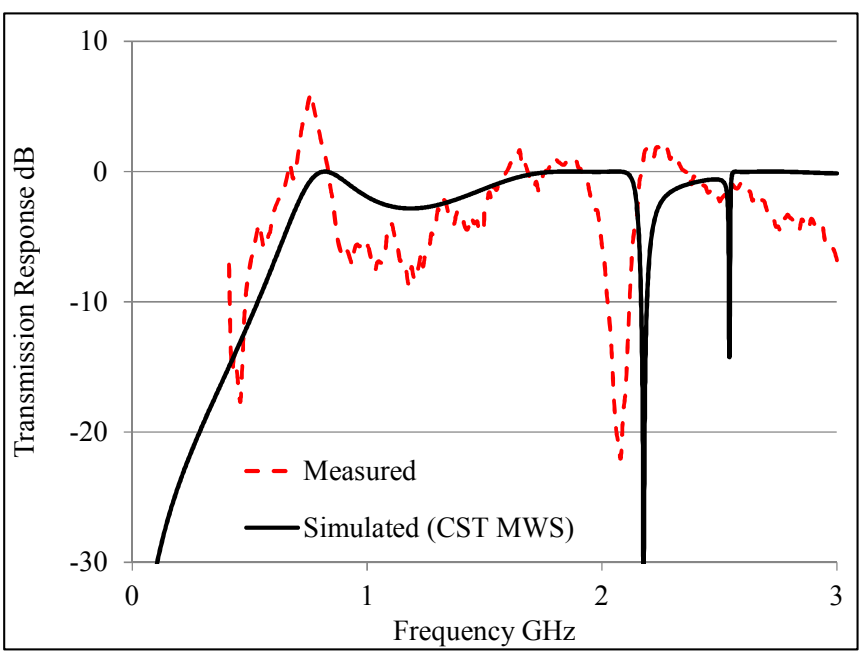

Fig. 10: Plane Wave Transmission Response of Double-Layer Grid Filter

[3] J. Batchelor, E.A. Parker, B. Sanz-Izquierdo, J.-B Robertson, and I. Ekpo, "Designing FSS for wireless propagation control within buildings", Antennas \& Propagation Conference, 2009. LAPC 2009. Loughborough, pp 14-17

[4] Callaghan, P., Parker, E.A. \& Langley, R.J.: 'Influence of supporting dielectric layers on the transmission properties of frequency selective surfaces', IEE Proc., Pt. H, Vol.138, No.5, Oct 1991, pp448-454

[5] B. Sanz-Izquierdo, E. A. Parker, J.-B. Robertson, and J. C. Batchelor, "Singly and dual polarized convoluted frequency selective structures," IEEE Trans. Antennas Propag., vol. 58, no. 3, pp. 690-696, Mar. 2010

[6] F. Deng ; X. Yi \& W. Wu: 'Design and Performance of a DoubleLayer Miniaturized-Element Frequency Selective Surface', IEEE Antennas and Wireless Propagation Letters, Vol. 12, 2013 , pp 721724

[7] S.I. Sohail, K.P. Esselle, G Kiani, "Design of a bandpass FSS on dual layer energy saving glass for improved RF communication in modern buildings", IEEE Antennas and Propagation Society International Symposium (APSURSI), 2012, pp1-2

[8] I-G Leeo, I-P. Hong: 'Frequency Selective Surface Design for Blinds Applications', 7th European Conference on Antennas and Propagation, 2013, pp 2753-2755

[9] N. Qasem \& Rob Seager; 'Overcoming the influence of people shadowing and enhancing MIMO capacity systems via modified environments', Antennas \& Propagation Conference, 2012. LAPC 2012. Loughborough, pp

[10] C. Christodoulou, D. P. Kwon, R. Middleveen and P.F. Wahid: 'Scattering from stacked gratings and dielectrics for various angles of wave incidence', IEEE Trans Vol. TAP 36, No. 10 October 1988 pp 1435-1441

[11] Rudge Milne Olve and Knight: 'The handbook of Antenna Design', Published Peter Peregrinus, 1986, ISBN 0864310529, Ch. 14, pp11561157

[12] N Marcuvitz: 'Waveguide Handbook', Published Peter Peregrinus, 1993, ISBN 0863410588, Ch 5, pp284-285

[13] C. Thajudeen, A. Hoorfar, and F. Ahmad: 'Measured complex permittivity of walls with different hydration levels and the effect on power estimation of TWRI target returns', Progress In Electromagnetics Research B, Vol. 30, 2011, pp177-199 